\title{
Mitigation of the Effects of Unknown Sea Clutter Statistics by Using Radial Basis Function Network
}

\author{
Bojan VONDRA, Davor BONEFACIC \\ Dept. of Wireless Communications, Faculty of Electrical Engineering and Computing, University of Zagreb, \\ Unska 3, 10000 Zagreb, Croatia \\ bojan.vondra@fer.hr, davor.bonefacic@fer.hr \\ Submitted May 15, 2019 / Accepted February 18, 2020
}

\begin{abstract}
In this paper, we investigate feasibility of employing Radial Basis Function (RBF) network into non-coherent detection process, for detection of targets embedded in sea clutter of unknown statistics. We particularly have in mind Croatian part of Adriatic Sea, the local sea whose clutter statistic properties are not available in open literature. Performance of the detection process employing proposed RBF network is tested with simulated clutter samples based on real sea clutter data. These data were collected under sea state conditions that represent two thirds of the total wave heights in Adriatic and are chosen to represent unknown clutter statistics due to the fact that no single probability density function equally well fits amplitude distribution of the range bins under test. It is demonstrated that, compared to the traditional $[z \log (z)]$ method, RBF network with just four components and lognormal basis function, yields operating characteristics that better match designed ones.
\end{abstract}

\section{Keywords}

Sea clutter, Adriatic sea, lognormal, non-coherent detection, RBF, $[\mathrm{z} \log (\mathrm{z})]$ method

\section{Introduction}

Today, there is a growing need to increase a coastline safety by monitoring gaps of inadequate coverage of the principle radars for the illegal vessels, dim and highly maneuvering by assumption, embedded in extensive sea clutter. In Croatia, there is intention to fill these gaps with lightweight and Commercial Off-The-Shelf (COTS) radar sensors, installed on Unmanned Surface Vehicles (USV). These sensors are mostly impulse radars, with mechanical scanning antenna and logarithmic receiver, operating in X-frequency band and outputting only measured intensity of returned echoes. Thus, mitigation of clutter effect is commonly performed after envelope detection usually applying threshold that relies on clutter amplitude samples. To apply this process in a full scale, accurate clutter amplitude statistics should be known, but usually, samples are used to estimate parameters of assumed (or prior) model. Traditionally, this model is compound Gaussian process of the fast time varying component and the slow time varying texture with gamma distributed intensity (or radar cross section) [1]. While the fast varying component is due to scattering from the small capillary waves (wavelength on the order of centimeter or less, with surface tension as restoration force, generated by a local wind near the surface), the slow time varying component is due to reflection from the larger gravity waves (sea or swell), with the wavelength on the order of meter or above. These waves, whose restoration force is gravity, are generated by a stable wind, distant in the case of swell and local in the case of sea. But in some cases, measured amplitude of the sea returns shows larger distribution tail than expected by the model of gamma distributed texture intensity. To fit data better, other distributions of texture intensity were introduced, such as inverse gamma [2], [3] and inverse Gaussian [4]. These distributions account for the so-called spiky returns, events primarily associated with the breaking waves [5], which affect tail, giving rise to a higher probability of false detection.

The development presented in this paper mostly concerns the Adriatic Sea which is small and enclosed sea. Therefore, it can be assumed that the clutter statistics will be significantly different in comparison to the one commonly observed for the ocean. For the Adriatic it is specific that surface wind waves are limited by fetch and wind duration, so it is mostly immature sea and waves are for this reason steeper, with shorter wavelength than their counterparts in ocean. Moreover, existence of more than 1300 islands greatly affects local wind and wave characteristics and sea depths can not be neglected in the most of the basin. During winter, dominant winds are bura (italian bora), northnorthwest to east-northeast wind and jugo (sirocco family), east-southeast to south-southeast wind. Bura blows transversely over the Dinaric Mountains which shape its focus points. Usual wind speeds are up to $20 \mathrm{~ms}^{-1}$ but can exceed $50 \mathrm{~ms}^{-1}$. Development of bura wind waves is limited by relatively narrow fetch across the Adriatic, so the maximum wave heights are expected in range from $6.2 \mathrm{~m}$ to $7.2 \mathrm{~m}$. Opposed to waves generated by bura, waves generated by jugo are higher and longer and already formed enter through 
Otranto Strait, causing more developed sea state. In stormy conditions, wind speed of jugo can reach $30 \mathrm{~ms}^{-1}$ and maximum recorded wave height during this wind was $10.8 \mathrm{~m} \mathrm{[6].}$

To the best of author's knowledge, measured clutter data and its statistics, particularly of Croatian part of the basin, do not exist in open literature. While one can expect that, qualitatively, behavior of the sea clutter returns follow statistics of measured data for fully developed sea, e.g. one can expect that shape parameter of amplitude distribution (which determines spikiness of the sea returns), is getting lower as radar resolution increases or grazing angle decreases, is lower for horizontal polarization and depends more on wind than on sea direction and slightly increases with increasing surface roughness (or increased sea state) [7], quantitative manifestation, i.e. statistics of returns depending on the sea state, significant wave height, wind speed, etc., are not known. For example, sea condition during measurements reported in [8], taken under short fetch condition (approximately $10 \mathrm{~km}$ ), can be thought as similar to Adriatic. In mentioned work, vertical polarization clutter data, recorded outside Toulon (France), in the Mediterranean, and in Taranto bay (southern Italy), were analyzed and compared to measurements and models derived for the fully developed ocean. For some trials it was found that model with inverse gamma distributed texture intensity better fits measured data than the one with gamma distribution. In contrast to measurements conducted under fully developed ocean as reported in [9], it follows that under similar wind conditions, shape parameter of the fitted distribution is not showing dependence on azimuth angle and is generally higher.

In this paper, performance of a non-coherent detection process employing non-parametric clutter model is investigated. Non-parametric model is built from the clutter samples only, without inclusion of any prior information about clutter. This is particularly important when the clutter statistics is unknown and target is dim. Any model mismatch in this case certainly leads to degradation of detection performance. Also, a non-parametric clutter model plays significant role in track-before-detect approach [10], where clutter (and target) statistics should be accurately evaluated for the fair tracker performance. So, aim of this paper is to investigate whether the change of paradigm from the prior clutter model (that may not approximate real clutter statistics well and thus cause processing losses) to non-parametric model (built entirely from the clutter samples), is feasible in non-coherent detection process. Such model should not be limited to stationary clutter only and should override shortcomings of model mismatch. In particular, target tracking method employing such model should be robust, in a sense that, compared to a traditional method that relies on prior clutter model, its performance should not be significantly worse when prior model (in traditional method) describes real clutter manifestation well, but should be significantly better when it does not. To build such model, we take advantage of the universal approximation property of neural networks, particularly RBF network [11], applied to the specific problem of density estimation.
There are numerous examples of using neural networks in density estimation. In context of radar application, for example in [12], RBF network was used to estimate clutter and target amplitude probability densities online. The primary reason of using RBF instead of Kernel Density Estimation (KDE) network in latter case lies in the fact that KDE often results in "bumpy" estimation, especially at the distribution tail which is not acceptable, as the tail is important in deriving detection threshold. Increasing width of the kernel function, which acts as the regularization parameter, smooths the estimate, but important information contained in the distribution tail is lost. In [13], neural network, based on recorded data, was used to estimate parameters of the Ricean Inverse Gaussian (RiIG) amplitude distribution (which can not be obtained in a closed form) and estimates were further used to set detection threshold assuming this particular clutter model. Neural network was also successfully used to reduce clutter in a conventional non-coherent radar system [14].

Similar recent approaches applied in detection process can be found in [15], where detection of small objects, distributed over few resolution cells and embedded in clutter, was based on estimation of the clutter amplitude probability density, employing KDE method [16]. Another example of using KDE network in detection process can be found in [17], where network, employing different kernels, was trained offline with real clutter data. Other examples of employing neural network in detection process include approaches of learning clutter statistics from the measured data online, or training to the various expected statistics offline, e.g. [18], where RBF network, based on available clutter data, was used to model nonlinear dynamics of the sea clutter by considering it as a chaotic system. In [19], RBF and Multi Layer Perceptron (MLP) networks were used, trained with different K-distribution shape parameters and Signal to Clutter Ratios (SCR), believed to be a good description of expected clutter and signal statistics. In [20], sea-ice clutter data were analyzed and used to train neural network with the result that such trained network, applied in coherent detection of non-fluctuating target echo embedded in Weibull sea-ice clutter, demonstrated enhanced detection and robustness compared to a conventional approximation of optimum NeymanPearson likelihood ratio detector. Amplitude statistics of the returned echoes and their temporal correlations were used in shore-based and non-coherent surveillance radar for detection of land areas and their exclusion from monitoring. MLP neural network was employed for classification of the each resolution bin echo as return from either land or sea [21]. Amplitude statistics of land and sea returns were modeled as Weibull and K-distributed, respectively. Interesting approach in non-coherent detection of a dim and sea surface target is given in [22], where detection of such a target was enhanced by employing Convolutional Neural Network (CNN). Network was trained by sequence of two dimensional images (range bin versus pulse number), in which position of a dim target echo was labeled. Thus trained network extracts essential features of sea clutter and target echo, making detection 
probability higher than in conventional Constant False Alarm Rate (CFAR) methods. In [23], detection of specific target among group, in background of sea clutter, was performed on the recognition basis, applying neural network and support vector machine with RBF kernel on high-resolution range profile. Network and support vector machine were trained with generated target range profiles and generally, support vector machine showed higher recognition rates under low signal to clutter ratios. In [24], detection of target with unknown Doppler shift, embedded in sea clutter, was improved (in comparison to conventional Doppler-preprocessed CFAR), by application of MLP neural network, trained with generated clutter patterns. Application of MLP neural network reduced clutter power variation and thus maintained fixed threshold value applied to conventional non-coherent CFAR detector. Advantage of this approach is that it has no restrictions on input clutter statistics. Conducted experiment on real sea clutter data showed, in comparison to conventional Doppler-preprocessed CFAR detector, better match to the designed false detection probability, thus, this approach is better approximation to Neyman-Pearson likelihood ratio detector.

In contrast to methods described in preceding paragraph, in this paper we use online learning of the clutter statistics. Similar to [15], the proposed method is not restricted to clutter statistics and does not require offline training or prior clutter model as in [19-21] or clutter samples as in [18,22-24]. In a more wider context of application of the proposed detection scheme in target tracking process, this is due to application of external association mechanism that separates set of samples into target echo and set of sea clutter echos, like in [12], where Viterbi based association scheme acts as target from clutter separator. This scheme is particularly suitable when targets are not dense, i.e. there is no overlapping of tracking gates between tracked targets.

Main contributions of this paper are the analysis of non-parametric clutter model accuracy and the performance of proposed non-coherent detector employing such model. Accuracy is derived based on theoretical probability distribution fit on data from illustrative real clutter example and performance is derived based on distribution's parameters of such fit.

This paper is organized as follows. In the next section, we briefly introduce RBF network as density estimator. We use such form of neural network to approximate unknown clutter amplitude distribution and employ it in noncoherent detection process. In the first part of Sec. 3, we introduce real sea clutter data from the medium resolution IPIX radar [25], [26] and use it as an illustrative example for testing proposed detection scheme. In the reminder of Sec. 3, performance comparison between employment of RBF network as non-parametric model and traditional $[\mathrm{z} \log (\mathrm{z})]$ estimator [27], [28] in detection process is presented. Finally, concluding remarks and outline of future work is given in Sec. 4.

\section{RBF Network as Density Estimator}

As already mentioned in the Introduction, unknown clutter density is estimated entirely from the samples and represented in functional form by a sum of some basis densities (or components). Using such an approximation is allowed as any bounded continuous probability density can be approximated by a finite sum of component densities, to any degree of accuracy [29], which is consistent with the universal approximation property of RBF network [11] as density estimation can be viewed as a special case of function approximation. One of the advantages of RBF network over non-parametric method, such as KDE, is the possibility of compacting information by grouping samples with similar statistics into same group. By putting density estimation into classification framework like in [30], parameters of the network can be determined by a maximum likelihood approach, which can be thought as a self-organizing method.

In [31], two main issues related to density estimation using sum of component densities were addressed. First was the quality of approximation, i.e. how well a certain class of basis densities can approximate unknown density, and second one was the quality of estimation, i.e. dependence of estimator performance on data set size. From [31], it follows that, given data size and a certain class of basis densities with a method of estimating their parameters, the total expected estimation error is bounded with a term related to adequacy of basis functions to approximate unknown density and a term related to the impact of fixed data size on the estimator. This can be expressed using expectation of squared Hellinger distance $d_{\mathrm{H}}^{2}$ between true density $p$ and its maximum likelihood approximation $\hat{f}_{n}^{\theta}$ (constituted from the sum of $n$ basis densities), as in [31],

$$
E\left(d_{\mathrm{H}}^{2}\left(p, \hat{f}_{n}^{\theta}\right)\right) \leq \epsilon+O\left(\frac{C}{n}\right)+O\left(\frac{m}{N}\right)
$$

where accuracy measure $\epsilon$ depends on class $\mathcal{F}$ of basis densities constituting $\hat{f}_{n}^{\theta}$, positive constant $C$ depends on both the true density and the class $\mathcal{F}, m$ depends on maximum likelihood estimator performance and $N$ is the number of samples used to evaluate $\hat{f}_{n}^{\theta}$. In contrast to commonly used KullbackLeibler divergence as distance measure between true density and its approximation, squared Hellinger distance [31]

$$
d_{\mathrm{H}}^{2}(p, f)=\int(\sqrt{p(\mathbf{x})}-\sqrt{f(\mathbf{x})})^{2} \mathrm{~d} \mathbf{x}, 0 \leq d_{\mathrm{H}}^{2} \leq 1,
$$

satisfies triangular inequality, thus allowing separation of error terms in (1).

From the classification point of view, each one of the $n$ basis densities can be thought as representative of some class $\omega$, hence, for some $l$-dimensional input $\mathbf{x}$, general approximation $f_{n}^{\theta}$ is given as

$$
f_{n}^{\theta}(\mathbf{x})=\sum_{i=1}^{n} f_{\sigma}\left(\mathbf{x} \mid \omega_{i} ; \boldsymbol{\theta}_{i}\right) P\left(\omega_{i}\right)
$$


where basis density $f_{\sigma}$ is radially symmetric and unimodal function, parametrization of generic low-bounded density $f$,

$$
f_{\sigma}(\mathbf{x}) \equiv \sigma^{-l} f\left(\frac{\|\mathbf{x}-\boldsymbol{\mu}\|}{\sigma}\right)
$$

where $\|\cdot\|$ is Euclidian norm, $\boldsymbol{\mu}$ vector of centers and $\sigma$ scaling. In (3), probability $P\left(\omega_{i}\right)$ is the prior probability of $i$ th class occurrence, and hence, $\sum_{i=1}^{n} P\left(\omega_{i}\right)=1$. Total set of parameters is given as $\boldsymbol{\theta}=\left\{\left\{P\left(\omega_{i}\right)\right\},\left\{\boldsymbol{\theta}_{i}\right\}, i=1, \ldots, n\right\}$, where $\boldsymbol{\theta}_{i}=\left(\boldsymbol{\mu}_{i}, \sigma_{i}\right)$. Since same scaling $\sigma$ is used across all dimensions, a prewhitening of data is required if the number of dimensions $l>1$.

Given input set of samples $\mathcal{X}=\left\{\mathbf{x}_{j}, j=1, \ldots, N\right\}, \log$ arithmic likelihood is defined as

$$
\ell(\boldsymbol{\theta} \mid \mathcal{X})=\frac{1}{N} \sum_{j=1}^{N} \log \left(f_{n}^{\theta}\left(\mathbf{x}_{j}\right)\right) .
$$

Maximization of (5) with respect to parameters $\boldsymbol{\theta}$,

$$
\hat{\boldsymbol{\theta}}_{N}=\arg \max _{\boldsymbol{\theta}}(\ell(\boldsymbol{\theta} \mid \mathcal{X}))
$$

yields $\hat{f}_{n}^{\theta}$ as maximum likelihood approximation of true density $p$.

Equation (3) is model of RBF network and its parameters can be determined by solving (6) which is done efficiently if we treat this problem in classification framework. Since samples as learning data are not labeled, a set of random variables $\mathcal{Z}=\left\{\mathbf{z}_{j}, j=1, \ldots, N\right\}$ is introduced, where each vector $\mathbf{z}_{j}=\left(z_{j 1}, \ldots, z_{j n}\right)$ indicates which sample is generated by which component $\left(z_{j i}\right.$ has value either 0 or 1$)$. Thus, $\mathcal{Z}$ can be treated as missing data in Expectation-Maximization (EM) algorithm and (5) can be rewritten as [32]

$$
\ell(\boldsymbol{\theta} \mid \mathcal{X}, \mathcal{Z})=\frac{1}{N} \sum_{j=1}^{N} \sum_{i=1}^{n} z_{j i} \log \left(f_{\sigma}\left(\mathbf{x}_{j} \mid \mathbf{z}_{j} ; \boldsymbol{\theta}\right) P\left(\mathbf{z}_{j} ; \boldsymbol{\theta}\right)\right)
$$

and solved by iteration. As shown in [33], at $k$ th step, expectation becomes

$$
Q\left(\boldsymbol{\theta} \mid \boldsymbol{\theta}_{k}\right)=E\left(\ell(\boldsymbol{\theta} \mid \mathcal{X}, \mathcal{Z}) \mid \mathcal{X}, \boldsymbol{\theta}_{k}\right),
$$

and the parameter $\boldsymbol{\theta}_{k+1}$ obtained by maximization is

$$
\boldsymbol{\theta}_{k+1}=\arg \max _{\boldsymbol{\theta}}\left(Q\left(\boldsymbol{\theta} \mid \boldsymbol{\theta}_{k}\right)\right) .
$$

The values obtained from (8) and (9) are values for the next iteration step. For generic Gaussian basis density

$$
f(\mathbf{x})=\frac{1}{(2 \pi)^{l / 2}} \exp \left(-\frac{\mathbf{x}^{2}}{2}\right)
$$

from (4) follows

$$
f_{\sigma}(\mathbf{x})=\frac{1}{(2 \pi)^{l / 2} \sigma^{l}} \exp \left(-\frac{\|\mathbf{x}-\boldsymbol{\mu}\|^{2}}{2 \sigma^{2}}\right),
$$

and EM algorithm ensures global convergence since generic density (10) belongs to class of exponential functions [31]. Expectation step performs $E\left(z_{j i} \mid \mathbf{x}_{j}, \boldsymbol{\theta}_{k}\right)$, resulting in posterior probability that $i$ th component has generated $j$ th sample, or, following [32],

$$
P_{k+1}\left(\omega_{i} \mid \mathbf{x}_{j}\right)=\frac{P_{k}\left(\omega_{i}\right) \sigma_{i, k}^{-l} \exp \left(-\frac{\left\|\mathbf{x}_{j}-\boldsymbol{\mu}_{i, k}\right\|^{2}}{2 \sigma_{i, k}^{2}}\right)}{\sum_{\iota=1}^{n} P_{k}\left(\omega_{i}\right) \sigma_{\iota, k}^{-l} \exp \left(-\frac{\left\|\mathbf{x}_{j}-\boldsymbol{\mu}_{\iota, k}\right\|^{2}}{2 \sigma_{\iota, k}^{2}}\right)}
$$

where $i=1, \ldots, n$ and $j=1, \ldots, N$. By applying maximization and using (12), the centers are given as

$$
\boldsymbol{\mu}_{i, k+1}=\frac{\sum_{j=1}^{N} \mathbf{x}_{j} P_{k+1}\left(\omega_{i} \mid \mathbf{x}_{j}\right)}{\sum_{j=1}^{N} P_{k+1}\left(\omega_{i} \mid \mathbf{x}_{j}\right)},
$$

and scaling as

$$
\sigma_{i, k+1}^{2}=\frac{\sum_{j=1}^{N} P_{k+1}\left(\omega_{i} \mid \mathbf{x}_{j}\right)\left\|\mathbf{x}_{j}-\boldsymbol{\mu}_{i, k+1}\right\|^{2}}{\sum_{j=1}^{N} P_{k+1}\left(\omega_{i} \mid \mathbf{x}_{j}\right)} .
$$

Finally, posterior probability of class $\omega_{i}$ for the next iteration step $k+1$ is given as

$$
P_{k+1}\left(\omega_{i}\right)=\frac{1}{N} \sum_{j=1}^{N} P_{k+1}\left(\omega_{j} \mid \mathbf{x}_{j}\right) .
$$

Steps (12)-(15) are repeated until the logarithmic likelihood

$$
\ell(\boldsymbol{\theta} \mid \mathcal{X})=\frac{1}{N} \sum_{j=1}^{N} \log \left(\sum_{i=1}^{n} \frac{P\left(\omega_{i}\right)}{(2 \pi)^{l / 2} \sigma_{i}^{l}} \exp \left(-\frac{\left\|\mathbf{x}_{j}-\boldsymbol{\mu}_{i}\right\|^{2}}{2 \sigma_{i}^{2}}\right)\right)
$$

converges to its maximum.

\section{Practical Example}

As already reported in [2], in the case of excessive spiky returns there is evident difference in distribution tail among different texture intensity models. Consider a compound Gaussian process [1]. If texture intensity $\zeta$ is modeled with gamma distribution

$$
p_{\Gamma}(\zeta)=\frac{\zeta^{v-1}}{\left(\frac{\beta}{2}\right)^{v} \Gamma(v)} \exp \left(-\frac{2 \zeta}{\beta}\right),
$$

and fast fluctuating component with complex Gaussian process, with unity variance for in- and quadrature-phase components, resultant clutter model is the well-known K-distribution

$$
p_{\mathrm{K}}(x)=\frac{4 x^{v}}{\beta^{\frac{v+1}{2}} \Gamma(v)} K_{v-1}\left(\frac{2 x}{\sqrt{\beta}}\right)
$$

where $v$ and $\beta$ are shape and scale parameters, respectively. In (18), $\Gamma$ is gamma function and $K_{v}$ is modified Bessel function of the second kind [34]. Texture intensity for amplitude distribution with higher tail than K-distribution is often modeled with inverse gamma distribution as

$$
p_{\Gamma^{-1}}(\zeta)=\frac{\zeta^{-v-1}}{\left(\frac{\beta}{2}\right)^{v} \Gamma(v)} \exp \left(-\frac{2}{\beta \zeta}\right)
$$


where $v$ and $\beta$ are again shape and scale parameters respectively. Here, the resulting clutter model is given as

$$
p_{\sqrt{\mathrm{GP}}}(x)=\frac{x \beta \Gamma(v+1)}{2\left(\frac{\beta}{4} x^{2}+1\right)^{v+1} \Gamma(v)},
$$

for which its intensity distribution $p\left(y=x^{2}\right)$ is known as Pareto Type II distribution.

Example shown in Fig. 1, using real sea clutter data from the medium resolution IPIX radar [25], [26], under condition of sea states 3-4 and 4-5, illustrates the dependence of distribution tail on sea state and polarization. This example is chosen deliberately as these states were reported to occur at 43\% (state 3-4) and 17\% (state 4-5) of time in Adriatic basin, which is about two thirds of the total wave heights in Adriatic (ignoring sea states 0 and 1-2 which produce insignificant clutter returns) [35]. It is assumed that spikiness of this local sea will not be higher than presented with this example. While model $p_{\sqrt{\mathrm{GP}}}$ refers to sea state $3-4$, model $p_{\mathrm{K}}$ refers to sea state 4-5. Parameters of the depicted distributions are given in Tab. 1 and were estimated using maximum likelihood approach (in the case of $p_{\mathrm{K}}$ ) [36], and using [zlog(z)] method (for the $p_{\sqrt{\mathrm{GP}}}$ ) [27], neglecting thermal noise in both cases, which is valid, considering radar resolution of $0.9^{\circ}$ in azimuth and $30 \mathrm{~m}$ in range.

It should be noted that there is particular reason that motivated us in choosing this data as example. It is the fact that no single probability density function equally well fits amplitude distribution of the range bins under test. Thus, it is suitable to employ RBF form of neural network to approximate unknown clutter amplitude distribution. This complex behavior of clutter is in agreement with [8], as already discussed in the Introduction.

Set of numerical trials, conducted as extension of [12] but not yet published, reveals that probability of track loss of a dim and maneuvering surface target making a $0.5 \mathrm{~g}$ U-turn, is below $2 \times 10^{-1}$ for false detection probability lower than $1.5 \times 10^{-1}$, shape parameter of K-distributed clutter $v=10$ (which is nearly Rayleigh clutter) and single-hit detection probability of $8 \times 10^{-1}$. With false detection probability lower than $2 \times 10^{-2}$, track loss reaches its plateau region of about $3 \times 10^{-2}$. For the more heavy-tailed K-distributed clutter $(v=0.5)$, probability of track loss below $2 \times 10^{-1}$ is achieved with false detection probability lower than $1.3 \times 10^{-1}$ and probability of track loss lower than $3 \times 10^{-2}$ is achieved with false detection probability lower than $5 \times 10^{-2}$. Therefore, in this paper we are focused on range of false detection probabilities from $10^{-3}$ to $10^{-1}$. Furthermore, through remaining of this paper, it is assumed that clutter samples are collected employing Viterbi association scheme, described in [12] (for the more details see [37]), which acts as a target from clutter discriminator. Thus, under assumption of perfectly correct associations, clutter samples are not corrupted with target echo. Echoes from land are excluded from processing by means of a digital terrain map.

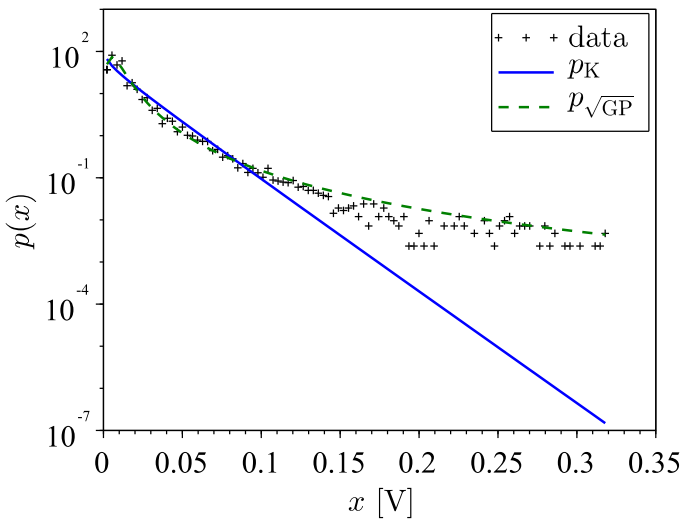

(a) Horizontal polarization, sea state $3-4$

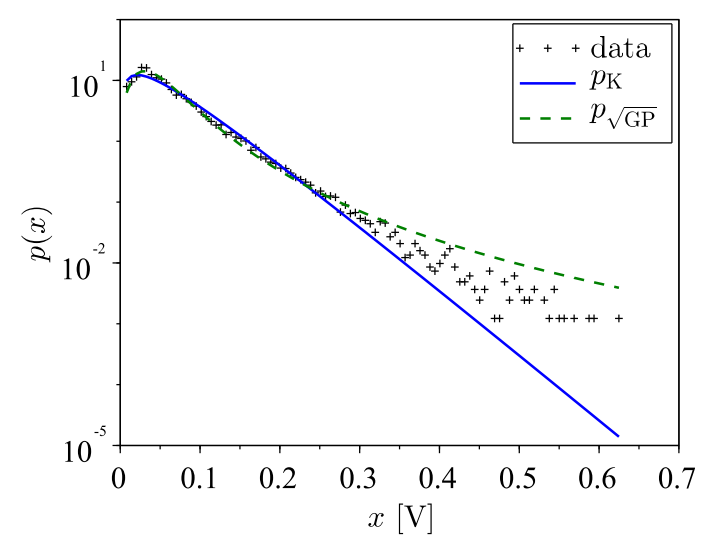

(b) Vertical polarization, sea state 4-5

Fig. 1. Difference in distribution tail and polarization when texture intensity of clutter is modeled with gamma distribution, resulting with density $p_{\mathrm{K}}(18)$, and inverse gamma distribution, resulting with density $p_{\sqrt{\mathrm{GP}}}(20)$.

\begin{tabular}{|c|c|c|c|c|}
\hline \multirow{2}{*}{ Sea state } & \multicolumn{4}{|c|}{ Distribution model } \\
\cline { 2 - 5 } & \multicolumn{2}{|c|}{$p_{\mathbf{K}}(18)$} & \multicolumn{2}{|c|}{$p_{\sqrt{\mathbf{G P}}}(20)$} \\
\cline { 2 - 5 } & Shape, $\boldsymbol{v}$ & Scale, $\boldsymbol{\beta}$ & Shape, $\boldsymbol{v}$ & Scale, $\boldsymbol{\beta}$ \\
\hline 3-4 (Fig. 1a) & 0.38 & 0.00109 & $\mathbf{1 . 0 2}$ & $\mathbf{4 9 6 7 8 . 1 9}$ \\
\hline 4-5 (Fig. 1b) & $\mathbf{1 . 0}$ & $\mathbf{0 . 0 0 6 2 0}$ & 1.53 & 1067.66 \\
\hline
\end{tabular}

Tab. 1. Estimated parameters of different sea clutter distributions depicted in Fig. 1. Parameters of better curve fit are emphasized.

\subsection{Network Design}

In the reminder of this subsection, we will determine the number of RBF network components. The number of network components depends on the desired accuracy, which depends on the number of samples that are in turn determined by the resolution of the radar system and any prior knowledge about spatial and temporal correlation of the clutter. Inspired by the example shown in Fig. 1, we use its data as input samples, with the parameters given in Tab. 1. As we do not have any prior knowledge about constant $C$ and estimator performance concentrated in $m$ (1), we will attempt to numerically derive the dependence of expectation of squared Hellinger distance (1) on (a): number of samples, given the fixed number of components and (b): on number 
of components, given the fixed number of samples. This is performed in both amplitude and logarithmic domain, where under term "amplitude domain", we mean that the $i$ th mixture component in (3) is given with Gaussian basis density

$$
f_{\sigma}\left(x \mid \omega_{i}, \boldsymbol{\theta}_{i}\right)=\frac{1}{\sigma_{i} \sqrt{2 \pi}} \exp \left(-\frac{\left(x-\mu_{i}\right)^{2}}{2 \sigma_{i}^{2}}\right),
$$

and under term "logarithmic domain", the $i$ th mixture component is given as lognormal basis density

$$
f_{\sigma}\left(x \mid \omega_{i} ; \boldsymbol{\theta}_{i}\right)=\frac{1}{x \sigma_{i} \sqrt{2 \pi}} \exp \left(-\frac{\left(\log (x)-\mu_{i}\right)^{2}}{2 \sigma_{i}^{2}}\right)
$$

which is Gaussian under transformation $y=\log (x)$. Parameters of the network were estimated using EM approach as described in Sec. 2, by iterating through steps (12)-(15) until convergence criterion was satisfied.

Difference among approximation classes is illustrated in Fig. 2. By analyzing (1), it can be seen that expectation of squared Hellinger distance decreases as the number of samples increases, but regarding number of components, it is not obvious that increasing their number would eventually lead to smaller approximation error. This is illustrated in Fig. 2b as saturation region which occurs for larger values of $n$. Generally speaking, for the considered type of clutter, the RBF network in logarithmic domain shows better approximation performance and saturation occurs for $n=4$ components with $N=1024$ samples. Figure 3 shows that the same can be expected for smaller number of samples.

Important property of detector is dependency of exact false detection probability (that results from the applied method) on designed one. How number of samples influences this property for proposed detector is summarized in Tab. 2 and Tab. 3 for few key values and is also illustrated in Fig. 4, which shows one typical realization of density estimation in logarithmic domain. It can be visualized from threshold values that, for less spiky clutter (Fig. 4a), probabilities of false detection using larger number of samples are lower than designed and using smaller number of samples, are closer to designed. For more spiky clutter (Fig. 4b), opposite is true. Here, exact false detection probability is given as $\hat{P}_{\mathrm{FA}}=\int_{T_{n}^{\theta}}^{\infty} \hat{f}_{n}^{\theta}(x) \mathrm{d} x$, and designed one as

$$
P_{\mathrm{FA}}=\int_{T}^{\infty} p(x) \mathrm{d} x
$$

where $p(x)$ is true clutter distribution, $\hat{f}_{n}^{\theta}(x)$ is its approximation and $T, T_{n}^{\theta}$ are corresponding threshold values. For application of proposed detector, scenario of more spiky clutter is more important than scenario of less spiky one, hence, through the rest of the paper, we will concentrate on larger number of samples.

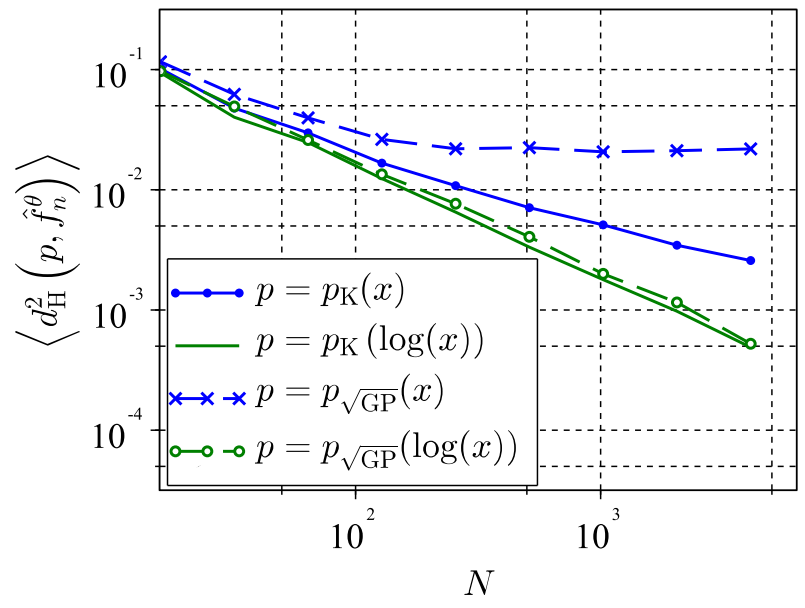

(a) Fixed number of components

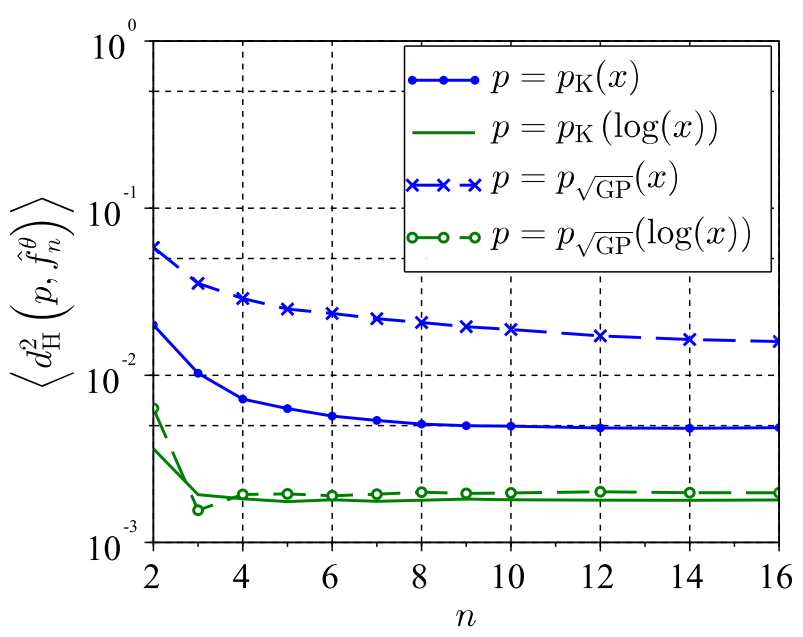

(b) Fixed number of samples

Fig. 2. Dependence of expectation of squared Hellinger distance on (a), number of samples $N$ with $n=8$ components and (b), number of components $n$ with $N=1024$ samples. Averaged over 100 runs.

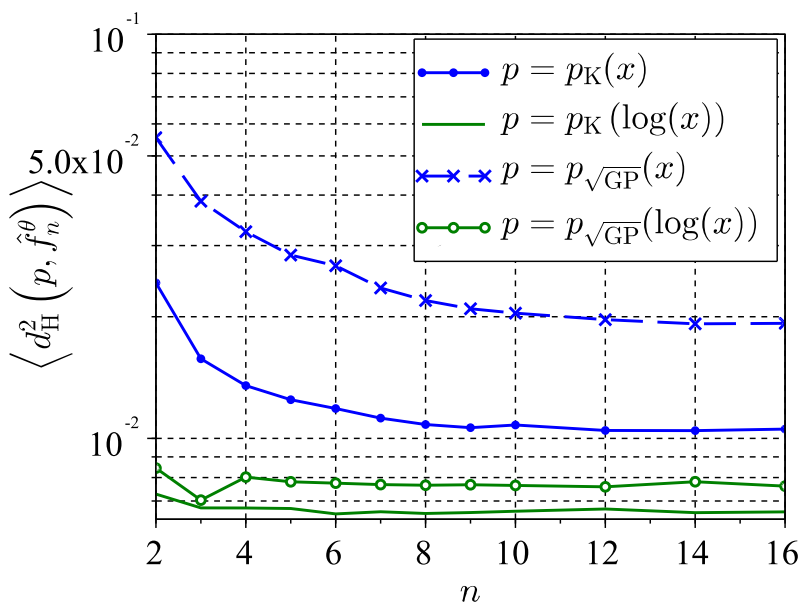

Fig. 3. Dependence of expectation of squared Hellinger distance on number of components $n$ with $N=256$ samples, averaged over 100 runs. 


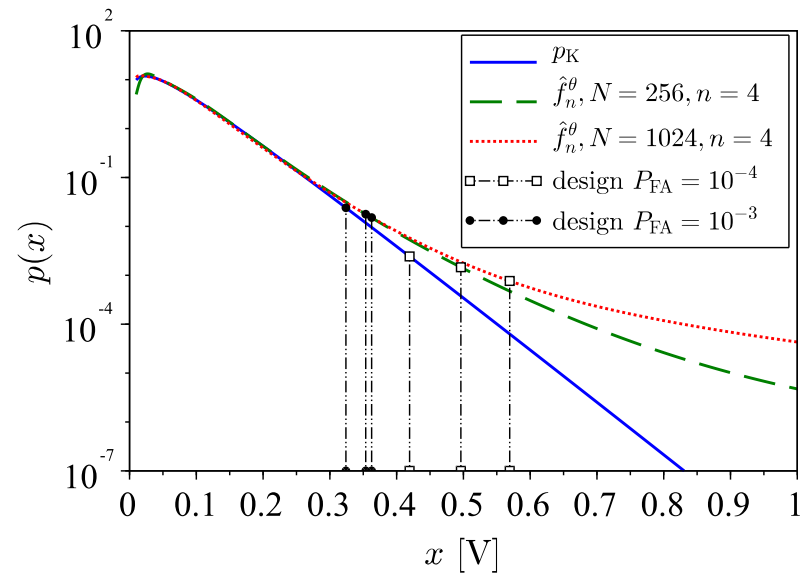

(a) Approximation of $p_{\mathrm{K}}$

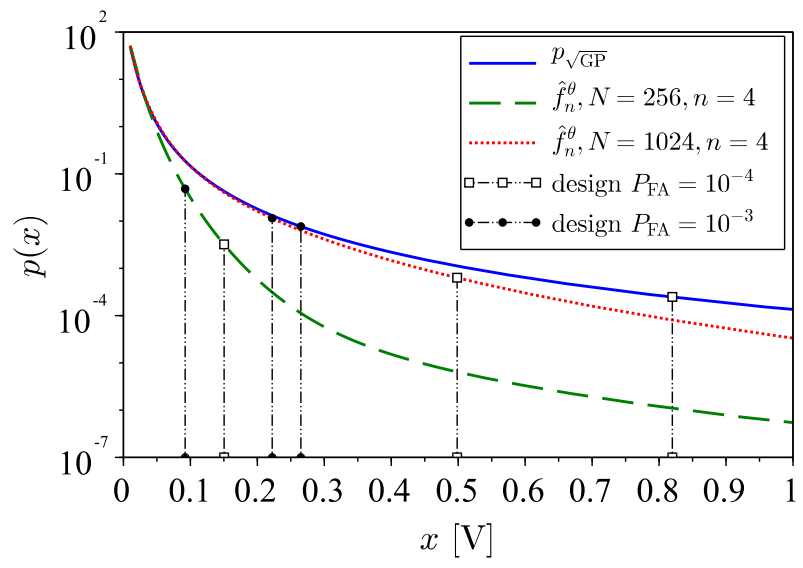

(b) Approximation of $p_{\sqrt{\mathrm{GP}}}$

Fig. 4. Comparison of various distributions approximation for single run and lognormal basis density. Threshold value corresponding to design false detection probability $P_{\mathrm{FA}}$ is shown as squares and dots, respectively.

\begin{tabular}{|c|c|c|}
\hline \multirow{2}{*}{ Design $\boldsymbol{P}_{\mathbf{F A}}$} & \multicolumn{2}{|c|}{ Exact $\boldsymbol{P}_{\mathbf{F A}}$ for distribution model $\boldsymbol{p}_{\mathbf{K}}(18)$} \\
\cline { 2 - 3 } & $\boldsymbol{N}=\mathbf{2 5 6}$ & $\boldsymbol{N}=\mathbf{1 0 2 4}$ \\
\hline $10^{-4}$ & $7.5 \times 10^{-5}$ & $4.3 \times 10^{-5}$ \\
\hline $10^{-3}$ & $9.6 \times 10^{-4}$ & $7.5 \times 10^{-4}$ \\
\hline $10^{-2}$ & $1.1 \times 10^{-2}$ & $9.9 \times 10^{-3}$ \\
\hline $10^{-1}$ & $1.0 \times 10^{-1}$ & $1.0 \times 10^{-1}$ \\
\hline
\end{tabular}

Tab. 2. Exact false detection probabilities for $p_{\mathrm{K}}$ distribution and two different numbers of samples $N$. Number of components is $n=4$. Lognormal basis density.

\begin{tabular}{|c|c|c|}
\hline \multirow{2}{*}{ Design $\boldsymbol{P}_{\mathbf{F A}}$} & \multicolumn{2}{|c|}{ Exact $\boldsymbol{P}_{\mathbf{F A}}$ for distribution model $\boldsymbol{p}_{\sqrt{\mathbf{G P}}}(20)$} \\
\cline { 2 - 3 } & $\boldsymbol{N}=\mathbf{2 5 6}$ & $\boldsymbol{N}=\mathbf{1 0 2 4}$ \\
\hline $10^{-4}$ & $8.4 \times 10^{-4}$ & $3.8 \times 10^{-4}$ \\
\hline $10^{-3}$ & $2.4 \times 10^{-3}$ & $1.5 \times 10^{-3}$ \\
\hline $10^{-2}$ & $1.2 \times 10^{-2}$ & $9.7 \times 10^{-3}$ \\
\hline $10^{-1}$ & $1.0 \times 10^{-1}$ & $1.0 \times 10^{-1}$ \\
\hline
\end{tabular}

Tab. 3. Exact false detection probabilities for $p_{\sqrt{\mathrm{GP}}}$ distribution and two different numbers of samples $N$. Number of components is $n=4$. Lognormal basis density.
When choosing number of samples, one must be aware of temporal and spatial correlation of the clutter. As already pointed out in [38], medium resolution radar with scanning antenna (such as conventional radar systems for maritime surveillance), results with texture temporal correlation of the order of the time-on-target and spatial correlation of the order of few tens of resolution range bins, provided that illuminated area is not highly irregular. If we denote a set of $L$ range bins, fixed in azimuth $j$, as

$$
\mathcal{X}_{j}=\left\{x_{i}, i=1, \ldots, L\right\},
$$

$N_{\rho}$ neighboring bins are correlated. Through the remaining of this subsection, we use the simplest form of correlation coefficients $\rho_{i, \iota}$, between range bins with indexes $i$ and $\iota$, both at azimuth $j$, as

$$
\rho_{i, \iota}=\left\{\begin{array}{l}
1,|i-\iota| \leq N_{\rho} \\
0,|i-\iota|>N_{\rho}
\end{array} .\right.
$$

Using $M$ such strips, the set of reference cells is finally given as

$$
\mathcal{X}=\left\{\mathcal{X}_{j}, j=1, \ldots, M\right\} .
$$

Therefore, upper value between eight to sixteen such strips, separated by radar resolution in azimuth, each with $N_{\rho}=16$ range bins, is usually adequate. If clutter is assumed stationary during several antenna scans, one can collect samples from several antenna scans and thus increase the number of samples. If $F$ such scans are used and if $\mathcal{X}^{i}$ denotes the set of samples at time $i$, the total set of samples $\mathcal{X}$ at current scan $k$ is given as

$$
\mathcal{X}=\left\{\mathcal{X}^{k-i}, i=0, \ldots, F-1:\left|X^{k-i}\right|=M L\right\}
$$

where each $\mathcal{X}^{k-i}$ in (27) corresponds to set (26). In this paper, we adopt samples from $F=4$ to $F=8$ successive antenna scans, resulting in total of $N=1024$ samples.

\subsection{Operating Characteristics}

For the purpose of mathematical tractability, optimum Neyman-Pearson likelihood ratio test [39], in the case of nonGaussian clutter, is usually replaced with some other nonoptimum, but sufficient test statistic that depends solely on assumed clutter model. However, under assumption of particular model, clutter can manifest differently due to model mismatch, causing detection losses and eventual track failures as model mismatch results in either too much clutter observations, or in detection threshold that is estimated higher than needed. In this work, we aim to approximate unknown clutter density with the sum of arbitrary probability functions whose statistics, and thus detection threshold, is known. This is similar to the reference method where parameters of the prior model are estimated from the clutter samples, and from the statistics of prior model with such estimated parameters, detection threshold is derived, see e.g. [40] for the K-distribution clutter model. 
Traditional approach in target detection is based on a binary hypothesis test, i.e. between two hypothesis $\mathrm{H} 0$ and $\mathrm{H} 1$ ( $\mathrm{H} 0$ that target is not present and $\mathrm{H} 1$ that target is present), one chooses $\mathrm{H} 0$ if the amplitude from the cell under test is below some threshold, determined by Type I error (choosing hypothesis $\mathrm{H} 1$ although $\mathrm{H} 0$ is true) and choosing hypothesis H1 otherwise [39]. From the constraint of Type I error, which is design parameter (in radar context, this is the probability of false detection $P_{\mathrm{FA}}$, given as $P_{\mathrm{FA}}=\int_{T}^{\infty} p(x) \mathrm{d} x$ where $p(x)$ is the probability density for H0), in the framework of density estimation by RBF network, false detection probability becomes

$$
P_{\mathrm{FA}}=\int_{T_{n}^{\theta}}^{\infty} \hat{f}_{n}^{\theta}(x) \mathrm{d} x=\sum_{i=1}^{n} \int_{T_{n}^{\theta}}^{\infty} P\left(\omega_{i}\right) f_{\sigma}\left(x \mid \omega_{i} ; \boldsymbol{\theta}_{i}\right) \mathrm{d} x,
$$

from which one can derive the detection threshold $T_{n}^{\theta}$.

In [41], detector for Pareto Type II clutter using sliding window based approach in Bayesian framework was proposed. Although true CFAR detector, it shows lower detection performance in comparison to techniques based on geometric transformation or ordered statistics that require prior knowledge of shape parameter in the former and scale parameter in the latter case [42]. Another drawback of the CFAR detector proposed in [41] is the need for numerical integration, in contrast to the method of moments which can be used in geometrical transformation and ordered statistics approach. Hence, for simplicity, in this paper we adopt traditional $[z \log (z)]$ method as the reference method for shape parameter estimation of both the Pareto Type II (this is density (20) under transformation $y=x^{2}$ ), and the K-distributed clutter (18), although it is difficult to capture statistics of such low values of shape parameter as given in example in Fig. 1.

Simulation flowchart is shown in Fig. 5 and its parameters, selected on the basis of emphasized parameters in Tab. 1, are shown in Tab. 4. Simulation was performed using tool for numerical computation Scilab [43], running on operating system openSUSE Leap 15.1 [44] and Intel "Ivy Bridge" processor platform [45]. It was divided into seven stages:

1. Initialization with parameters as per Tab. 4.

2. Evaluation of reference threshold $T$ and reference Signal to Clutter Ratio (SCR), depending on designed value of false detection probability $P_{\mathrm{FA}}$ and target detection probability $P_{\mathrm{D}}$. Given set of designed false detection probabilities as $\mathcal{P}_{\mathrm{FA}}=\left\{P_{\mathrm{FA}_{1}}, P_{\mathrm{FA}_{2}}, \ldots\right\}$, for each $P_{\mathrm{FA}}$ and given clutter distribution model $p$, threshold $T$ is calculated from (23), (18) and (20), as solution of

$$
P_{\mathrm{FA}}= \begin{cases}\frac{2 T^{v} K_{v}(2 T / \sqrt{\beta})}{\beta^{v / 2} \Gamma(v)}, & p=p_{\mathrm{K}} \\ 1-\frac{\Gamma(v+1)\left(\beta T^{2}+4\right)^{v}-4^{v} \Gamma(v+1)}{v \Gamma(v)\left(\beta T^{2}+4\right)^{v}}, & p=p_{\sqrt{\mathrm{GP}}}\end{cases}
$$

Next, one must find signal strength $S$ (target echo power) or equivalently SCR such that, given threshold $T$ as solution of (29), probability detection equals designed value $P_{\mathrm{D}}$. As expected sea targets are usually classified by Swerling Case 1 model [46], detection probability, in the case of gamma distributed texture intensity, is given as

$$
\begin{aligned}
P_{\mathrm{D}}= & \frac{1}{\left(\frac{\beta}{2}\right)^{\nu} \Gamma(\nu)} \int_{T}^{\infty} x \mathrm{~d} x \\
& \int_{0}^{\infty} \frac{\zeta^{\nu-2}}{1+\mathrm{SCR}} \exp \left(-\frac{2 \zeta}{\beta}-\frac{x^{2}}{2 \zeta(1+\mathrm{SCR})}\right) \mathrm{d} \zeta .
\end{aligned}
$$

Equation (30) is solved numerically using the approach given in [40]. In the case of inverse gamma texture intensity distribution, detection probability is given as

$$
\begin{aligned}
P_{\mathrm{D}}= & \frac{1}{\left(\frac{\beta}{2}\right)^{v} \Gamma(v)} \int_{T}^{\infty} x \mathrm{~d} x \\
& \int_{0}^{\infty} \frac{\zeta^{-v-2}}{1+\mathrm{SCR}} \exp \left(-\frac{2}{\beta \zeta}-\frac{x^{2}}{2 \zeta(1+\mathrm{SCR})}\right) \mathrm{d} \zeta
\end{aligned}
$$

which is solved using Gauss-Kronrod quadrature variant of Guassian quadrature method [47]. In this paper, we have chosen SCR such that designed detection probability $P_{\mathrm{D}}$ always equals $8 \times 10^{-1}$. For the gamma model of texture intensity, mean clutter power equals $v \beta / 2$ and for the inverse gamma model, it equals $\frac{4}{\beta(v-1)}$, conditioned on $v>1$. Therefore, signal strength (or target echo power) $S$, is related to SCR as

$$
\mathrm{SCR}= \begin{cases}\frac{2 S}{v \beta}, & p=p_{\mathrm{K}} \\ \frac{S \beta(v-1)}{4}, & p=p_{\sqrt{\mathrm{GP}}}\end{cases}
$$

3 . Generation of $N$ clutter samples, respecting clutter distribution model $p$, correlation parameter $N_{\rho}$ and correlation coefficient given with (25). Samples are given as

$$
\begin{aligned}
\mathcal{X} & =\left\{\mathcal{X}_{i}, i=1, \ldots, N / N_{\rho}\right\} \\
\mathcal{X}_{i} & =\left\{x_{\iota}: x_{\iota} \sim \mathcal{N}\left(0, \sqrt{\zeta_{i}}\right), \zeta_{i} \sim p, \iota=1, \ldots, N_{\rho}\right\}
\end{aligned}
$$

where $\mathcal{N}(\mu, \sigma)$ is normal (or Gaussian) distribution with mean $\mu$ and deviation $\sigma$ and $p$ is $p_{\Gamma}$ for the $\mathrm{K}$ distribution model (18) and $p_{\Gamma^{-1}}$ for model with the inverse gamma texture (20).

4. Clutter density estimation. Using $[z \log (z)]$ approach, the shape parameter is estimated from intensity samples $\mathcal{Y}=\left\{y_{i} \mid y_{i}=x_{i}^{2}, i=1, \ldots, N\right\}$. For the K-distributed clutter, neglecting thermal noise and using only one pulse, the $[\mathrm{zlog}(\mathrm{z})]$ method gives [27],

$$
\frac{1}{\hat{v}}=\frac{\langle\mathcal{Y} \log (\boldsymbol{Y})\rangle}{\langle\boldsymbol{Y}\rangle}-\langle\log (\boldsymbol{Y})\rangle-1,
$$

and the scale parameter follows from the first moment of intensity,

$$
\hat{\beta}=\langle\boldsymbol{Y}\rangle \hat{v}^{-1}
$$


If texture intensity is modeled as inverse gamma distribution, the distribution of amplitude intensity $y=x^{2}$ is known as Pareto Type II distribution, so $[\mathrm{z} \log (\mathrm{z})]$ method estimates shape parameter according to [27],

$$
\frac{1}{\hat{v}-1}=\frac{\langle\mathcal{Y} \log (\mathcal{Y})\rangle}{\langle\mathcal{Y}\rangle}-\langle\log (\mathcal{Y})\rangle-1, \hat{v}>1,
$$

again neglecting effects of thermal noise and using just one pulse. The above is valid for $\hat{v}>1$ and the scale parameter follows from the first moment of intensity as,

$$
\hat{\beta}=\frac{4}{\langle\boldsymbol{Y}\rangle}(\hat{v}-1), \hat{v}>1 .
$$

Parameters $\boldsymbol{\theta}$ of the RBF network were estimated using EM method in logarithmic domain (see Sec.2 and Sec.3.1), employing iteration steps (12) through (15). Also, a squared Hellinger distance $d_{\mathrm{H}}^{2}$ is outputted.

5. Detection thresholds. For the K-distribution (18), detection threshold is determined by solving $P_{\mathrm{FA}}=$ $\int_{T_{\mathrm{K}}}^{\infty} p_{\mathrm{K}}(x) \mathrm{d} x$ with (35) and (36) as input parameters. Detection threshold $T_{\sqrt{\mathrm{GP}}}$ is the solution of equation $P_{\mathrm{FA}}=\int_{T_{\sqrt{G \mathrm{P}}}}^{\infty} p_{\sqrt{\mathrm{GP}}}(x) \mathrm{d} x$, employing (37) and (38) as input parameters. Detection threshold $T_{n}^{\theta}$ for RBF network is determined by solving (28).

6. Operating characteristics. If one applies thresholds $T_{n}^{\theta}$, $T_{\mathrm{K}}$ and $T_{\sqrt{\mathrm{GP}}}$ (derived in Step 5) to true distributions (18) and (20), exact false and detection probabilities can be calculated for the scenario of less spiky and spiky clutter, for known SCR and for the matched and mismatched clutter model. This is done by defining threshold as function of prior clutter distribution model,

$$
g(T)= \begin{cases}T_{\mathrm{K}}, & p=p_{\mathrm{K}} \\ T_{\sqrt{\mathrm{GP}}}, & p=p_{\sqrt{\mathrm{GP}}} \\ T_{n}^{\theta}, & p=f_{n}^{\theta}\end{cases}
$$

and substituting it instead of $T$ in (29), (30) and (31).

7. In this stage, by means of sample averages of exact false and detection probabilities, evaluated over $J$ runs, we compare performance of the RBF network with the traditional method of equivalent distribution. Depicting exact false and detection probabilities versus designed ones, gives us idea about quality of approximation and feasibility of employing RBF network in

\begin{tabular}{|c|c|c|}
\hline Parameter & Description & Value \\
\hline$n$ & $\begin{array}{l}\text { Number of component } \\
\text { densities }\end{array}$ & 4 \\
\hline$J$ & Number of trials & 100 \\
\hline$N$ & Number of samples & 1024 \\
\hline$N_{\rho}$ & $\begin{array}{l}\text { Number of correlated } \\
\text { samples }\end{array}$ & 1 or 16 \\
\hline $\mathcal{P}_{\mathrm{FA}}$ & $\begin{array}{l}\text { Set of design false } \\
\text { detection probabilities }\end{array}$ & $10^{-4}-2 \times 10^{-1}$ \\
\hline$P_{\mathrm{D}}$ & Detection probability & $8 \times 10^{-1}$ \\
\hline$p$ & Clutter distribution model & $p_{\mathrm{K}}$ or $p_{\sqrt{\mathrm{GP}}}$ \\
\hline$v$ & Clutter distribution' shape & $\begin{array}{l}1.00 \text { for } p_{\mathrm{K}} \text { and } \\
1.02 \text { for } p_{\sqrt{\mathrm{GP}}}\end{array}$ \\
\hline$\beta$ & Clutter distribution' scale & $\begin{array}{l}0.0062 \text { for } p_{\mathrm{K}} \text { and } \\
49678.2 \text { for } p_{\sqrt{\mathrm{GP}}}\end{array}$ \\
\hline
\end{tabular}
detection process of target embedded in clutter with unknown statistics.
Tab. 4. Simulation parameters.

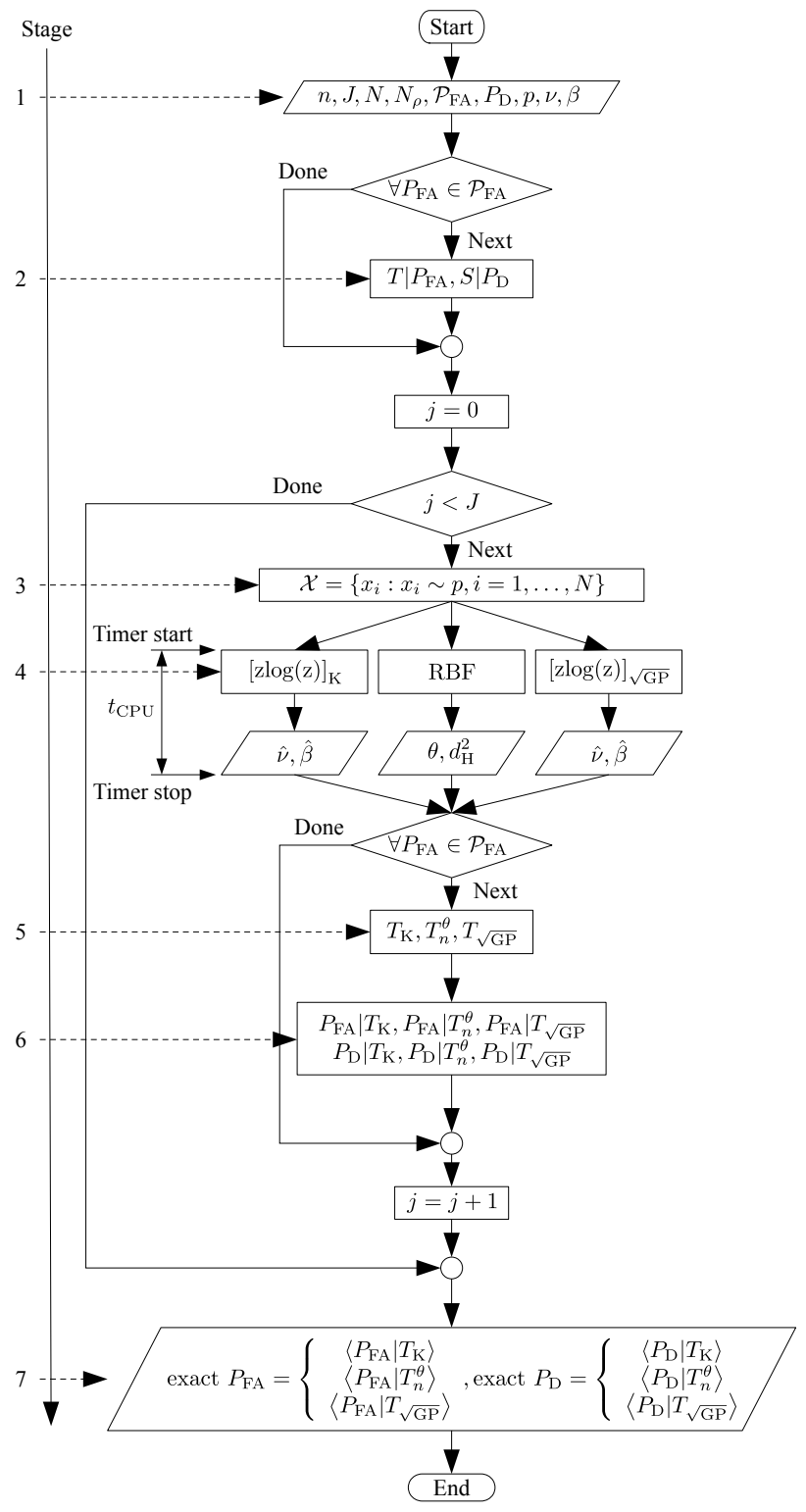

Fig. 5. Simulation flowchart. 


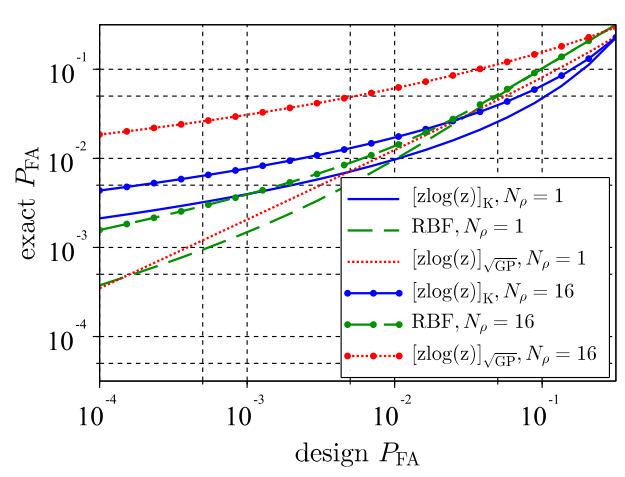

(a) False detection probability

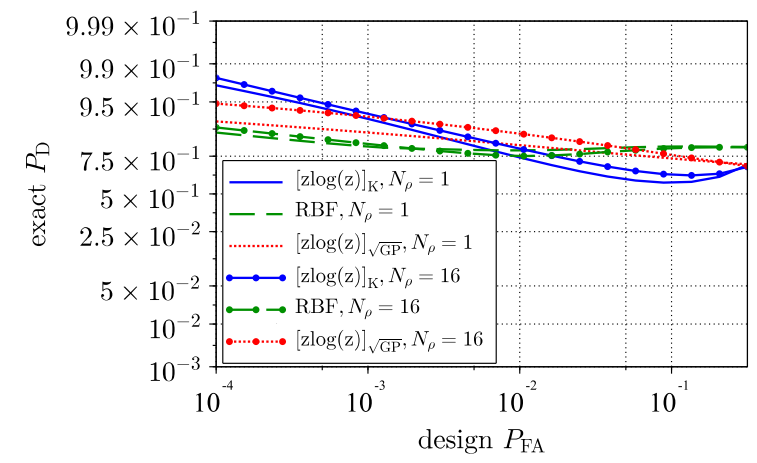

(b) Detection probability

Fig. 6. Performance of $[\mathrm{z} \log (\mathrm{z})]$ detection scheme with RBF network in logarithmic domain when texture intensity is modeled as inverse gamma distributed. This distribution corresponds to spiky clutter as shown in Fig. 1a.

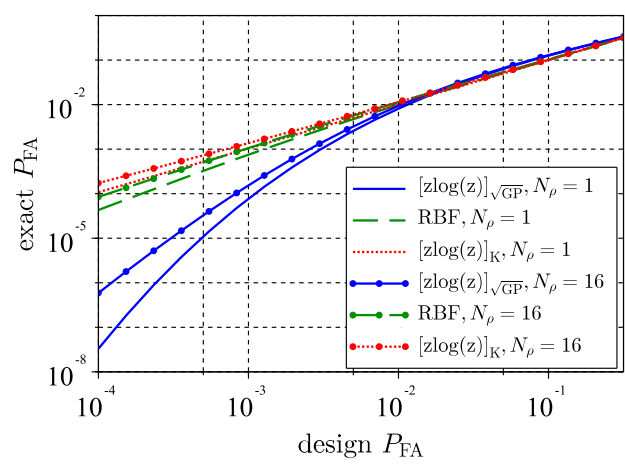

(a) False detection probability

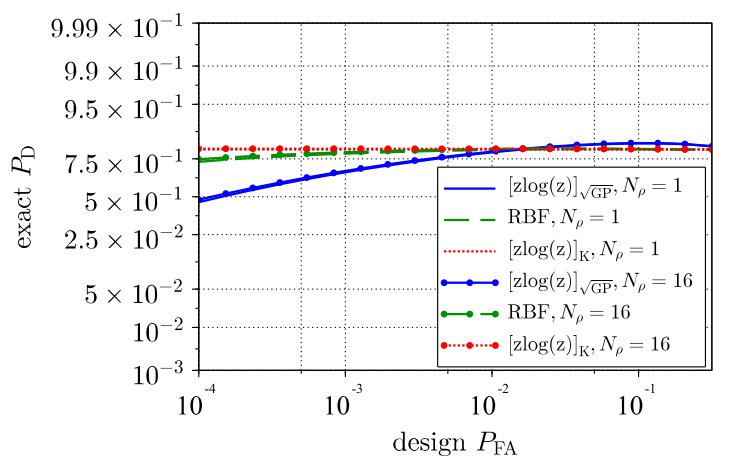

(b) Detection probability

Fig. 7. Performance of $[\mathrm{z} \log (\mathrm{z})]$ detection scheme with $\mathrm{RBF}$ network in logarithmic domain when texture intensity is modeled as gamma distributed. This distribution corresponds to less spiky clutter as shown in Fig. 1b.

Simulation results for the both $N_{\rho}=1$ and the $N_{\rho}=16$ correlated range bins are shown in Figs. 6 and 7 for the scenario of spiky and less spiky clutter, respectively. Exact false and detection probabilities are compared with the design ones, for the method which employs RBF network and for the $[z \log (\mathrm{z})]$ method which is either matched to the true distribution, or mismatched. While the index $\mathrm{K}$ emphasizes that the estimation of shape parameter is based on prior K-distribution according to (35) and (36), the index $\sqrt{\mathrm{GP}}$ emphasizes that estimation is based on prior Pareto Type II distribution of intensity, where parameters are estimated from (37) and (38). Results are based on average of 100 runs for each false detection probability point, 1024 clutter samples and lognormal basis density.

Results show that, even when the $[\operatorname{zlog}(z)]$ method matches true inverse gamma texture intensity distribution, there is significant deviation of exact false detection probabilities from designed one. This impacts the probability of detection, too. Furthermore, the effect of range bins correlation is more pronounced in the case of spiky clutter, which is manifested as increase of the false detection probability. Although the match is not perfect, it should be noted that, in the practical $P_{\mathrm{FA}}$ range from $10^{-3}$ onward, the RBF network better matches the designed $P_{\mathrm{FA}}$ than the $[\mathrm{z} \log (\mathrm{z})]$ methods, especially in the case of model mismatch. Drawback of this method is its computational load $\left(t_{\mathrm{CPU}}\right.$, refer to Stage 4 in the Fig. 5). Without calculation of the squared Hellinger distance and for the specified hardware and software configuration [43-45] (see Sec. 3.2), it takes on average $500 \mathrm{~ms}$ for the EM algorithm and $0.2 \mathrm{~ms}$ for the $[\mathrm{z} \log (\mathrm{z})]$ method which is about 2500 times faster. This is due to more intense calculation in steps (12)-(15) than in (35)-(38) and iterative nature of EM algorithm. On average, it takes about 100 iterations to achieve convergence of logarithmic likelihood (16) to the precision of $10^{-6}$.

The following discrepancy of $[\operatorname{zlog}(\mathrm{z})]$ method in the case of inverse gamma texture intensity can be explained with value of shape parameter that is very close to 1 , what is the limit of the estimator validity. To estimate this parameter with better accuracy, larger data sample size is required (e.g., for 1024 samples, the estimated shape parameter is 1.301 , for 4096 samples, 1.245, for 16384 samples, 1.201, and so on). On the other hand, if one is able to solve for the shape parameter using fractal moments, i.e. $\hat{m}_{1}=\langle x\rangle$ and $\hat{m}_{1 / 2}=\left\langle x^{1 / 2}\right\rangle$, and to solve the equation

$$
\frac{\hat{m}_{1}}{\hat{m}_{1 / 2}^{2}}=\frac{\Gamma(\hat{v}) \Gamma\left(\frac{3}{2}\right) \Gamma\left(\hat{v}-\frac{1}{2}\right)}{\left(\Gamma\left(\frac{5}{4}\right) \Gamma\left(\hat{v}-\frac{1}{4}\right)\right)^{2}}
$$

numerically [36], more accurate values of shape parameter would be estimated (i.e. the sample size 1024 gives 1.055 , while current method gives 1.301), but this imposes too high computational demands for real-time application and is beyond the scope of this paper. 


\subsection{Design Issue}

It may happen that, for the given design false detection probability and due to some excessive spike return as illustrated in Fig. 8, the estimated threshold value $T_{n}^{\theta}$ exceeds the dynamic range of the receiver. For this particular example, the designed false detection probability is $10^{-4}$ and the estimated threshold value is $T_{n}^{\theta} \approx 21 \mathrm{~V}$, which corresponds to the exact false detection probability $\approx 10^{-7}$. The RBF network is evaluated in logarithmic domain using $n=4$ components and $N=1024$ samples. One method to counteract this issue could be censoring of the maximum sample value. For this particular example, censoring gives $T_{n}^{\theta}=0.6 \mathrm{~V}$, resulting with the exact $P_{\mathrm{FA}}=1.9 \times 10^{-4}$ which is closer to the designed value. This is explained with better approximation of the true distribution tail when the censored data are employed. Nevertheless, the squared Hellinger distances are approximately the same (prior censoring 0.0021 and after censoring 0.0022). For this example, estimated probability densities prior and after censoring are illustrated in Fig. 9.

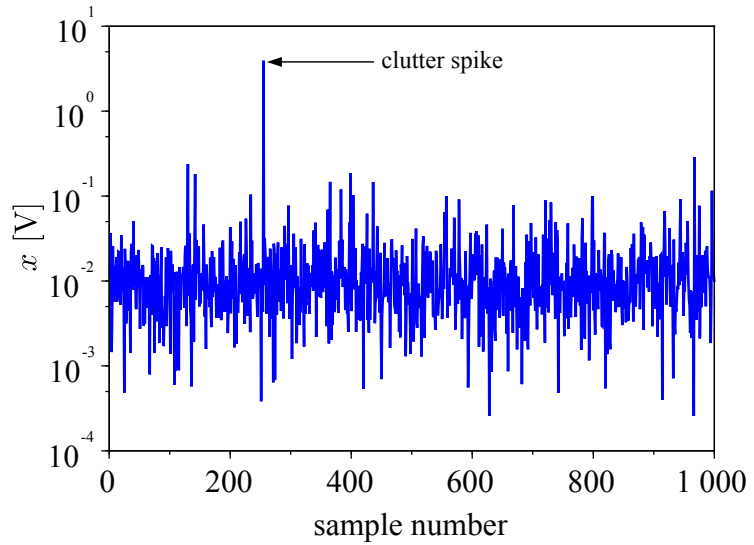

Fig. 8. Excessive clutter spike at sample number 255 and distribution $p_{\sqrt{\mathrm{GP}}}(20)$.

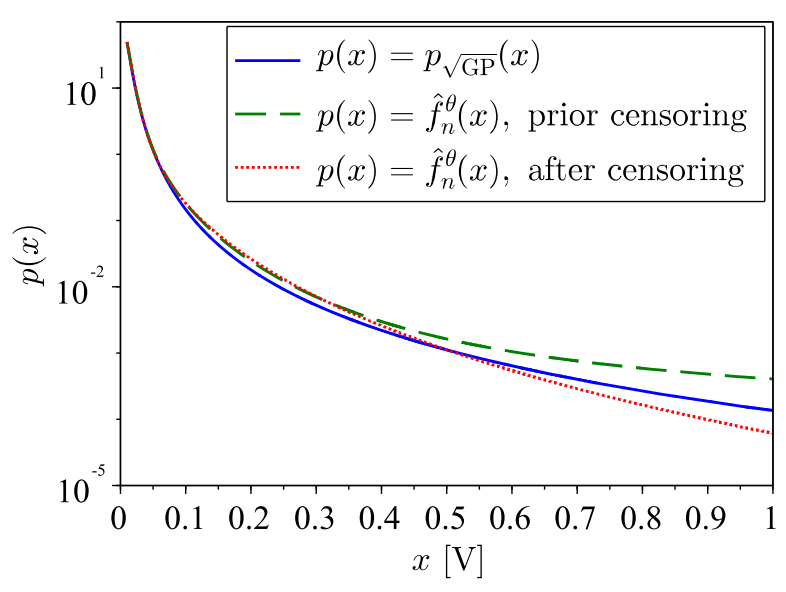

Fig. 9. True (blue solid line) and estimated probability densities prior (green dashed line) and after censoring (red dotted line). Number of samples is $N=1024$ and employed RBF network has $n=4$ components. Parameters of the network were estimated using EM method in logarithmic domain.

\section{Conclusion}

Although probability density estimation employing $\mathrm{RBF}$ network is not a new concept, in this paper it is tested in a new light of embedment in a non-coherent detection process, in a scenario when the sea clutter statistics is unknown. Performance analysis, conducted in Sec. 3.2, indicates that employment of RBF network in such a clutter yields receiver operating curves that are reasonably close to designed ones, closer than the ones obtained by the traditional $[\mathrm{z} \log (\mathrm{z})]$ method which uses estimating parameters of assumed (or prior) clutter model. It is also demonstrated that RBF with lognormal basis function better approximates spiky clutter than the one with the Gaussian function.

Future work will investigate the influence of the described clutter modeling when used in process of maneuvering target tracking and, in more challenging scenario, tracking a dim target using track-before-detect scheme. Such method often relies on clutter likelihood to improve tracking performance, as in [48] for the K-distribution prior model. Based on the presented results and some preliminary tests, it can be assumed that the employment of RBF network would offer larger improvement in comparison to the prior model in a scenario when the prior model does not match well the true statistics of clutter. This will also be addressed in our future work.

\section{References}

[1] GINI, F., GRECO, M. Texture modeling and validation using recorded high resolution sea clutter data. In Proceedings of the 2001 IEEE Radar Conference. Atlanta, GA (USA), 2001, p. 387-392. DOI: 10.1109/NRC.2001.923010

[2] MEZACHE, A., SOLTANI, F., SAHED, M., CHALABI, I. Model for non-Rayleigh clutter amplitudes using compound inverse Gaussian distribution: An experimental analysis. IEEE Transactions on Aerospace and Electronic Systems, 2015, vol. 51, no. 1, p. 142-153. DOI: 10.1109/TAES.2014.130332

[3] LEFAIDA, S., SOLTANI, F., MEZACHE, A. Radar seaclutter modelling using fractional generalised Pareto distribution. Electronics Letters, 2018, vol. 54, no. 16, p. 999-1001. DOI: $10.1049 / \mathrm{el} .2018 .5233$

[4] OLLILA, E., TYLER, D. E., KOIVUNEN, V., POOR, H. V. Complex elliptically symmetric distributions: Survey, new results and applications. IEEE Transactions on Signal Processing, 2012, vol. 60, no. 11, p. 5597-5625. DOI: 10.1109/TSP.2012.2212433

[5] MELIEF, H. W., GREIDANUS, H., VAN GENDEREN, P., et al. Analysis of sea spikes in radar sea clutter data. IEEE Transactions on Geoscience and Remote Sensing, 2006, vol. 44, no. 4, p. 985-993. DOI: 10.1109/TGRS.2005.862497

[6] KATALINIC, M., CORAK, M., PARUNOV, J. Maritime Technology and Engineering. London (UK): Taylor \& Francis Group, 2015. (Analysis of wave heights and wind speeds in the Adriatic Sea.) ISBN: 978-1-138-02727-5

[7] CRISP, D. J., ROSENBERG, L., STACY, N. J., et al. Modelling Xband sea clutter with the K-distribution: Shape parameter variation. In International Radar Conference "Surveillance for a Safer World". Bordeaux (France), 2009, p. 1-6. ISSN: 1097-5764 
[8] JOHNSEN, T. Characterization of X-band radar sea-clutter in a limited fetch condition from low to high grazing angles. In IEEE Radar Conference. Johannesburg (South Africa), 2015, p. 109-114. DOI: 10.1109/RadarConf.2015.7411864

[9] DONG, Y. Distribution of X-band high resolution and high grazing angle sea clutter. Research report DSTO-RR-0316, Defence Science and Technology Organisation (Australia), 2006, 88 pages. [Online] Cited 2019-04-27. Available at: http://cradpdf.drdcrddc.gc.ca/PDFS/unc56/p527164.pdf

[10] BERRY, P., VENKATARAMAN, K., ROSENBERG, L. Adaptive detection of low-observable targets in correlated sea clutter using Bayesian track-before-detect. In IEEE Radar Conference. Seattle (USA), 2017, p. 398-403. DOI: 10.1109/RADAR.2017.7944235

[11] PARK, J., SANDBERG, I. W. Universal approximation using radialbasis-function networks. Neural Computation, 1991, vol. 3, no. 2, p. 246-257. DOI: 10.1162/neco.1991.3.2.246

[12] VONDRA, B., BONEFACIC, D. Estimation of heavy-tailed clutter density using adaptive RBF network. In 22nd International Conference on Applied Electromagnetics and Communications (ICECom). Dubrovnik (Croatia), 2016, p. 1-6. DOI: 10.1109/ICECom.2016.7843876

[13] MEZACHE, A., CHALABI, I. Estimation of the RiIG-distribution parameters using the artificial neural networks. In IEEE International Conference on Signal and Image Processing Applications. Melaka (Malaysia), 2013, p. 291-296. DOI: 10.1109/ICSIPA.2013.6708020

[14] VICEN-BUENO, R., CARRASCO-ALVAREZ, R., ROSAZURERA, M., et al. Sea clutter reduction and target enhancement by neural networks in a marine radar system. Sensors, 2009, vol. 9, no. 3, p. 1913-1936. DOI: 10.3390/s90301913

[15] CUI, Y., YANG, J., YAMAGUCHI, Y., et al. On semiparametric clutter estimation for ship detection in synthetic aperture radar images. IEEE Transactions on Geoscience and Remote Sensing, 2013, vol. 51, no. 5, p. 3170-3180. DOI: 10.1109/TGRS.2012.2218659

[16] PARZEN, E. On estimation of a probability density function and mode. Annals of Mathematical Statistics, 1962, vol. 33, no. 3, p. 1065-1076. DOI :10.1214/aoms/1177704472

[17] ZHOU, H., LI, Y., JIANG, T. Sea clutter distribution modeling: a kernel density estimation approach. In 10th International Conference on Wireless Communications and Signal Processing (WCSP). Hangzhou (China), 2018, p. 1-6. DOI: 10.1109/WCSP.2018.8555876

[18] HENNESSEY, G., LEUNG, H., DROSOPOUlOS, A., et al. Seaclutter modeling using a radial-basis-function neural network. IEEE Journal of Oceanic Engineering, 2001, vol. 26, no. 3, p. 358-372. DOI: $10.1109 / 48.946510$

[19] CHEIKH, K., SOLTANI, F. Application of neural networks to radar signal detection in K-distributed clutter. IEE Proceedings Radar, Sonar and Navigation, 2006, vol. 153, no. 5, p. 460-466. DOI: 10.1049/ip-rsn:20050103

[20] VICEN-BUENO, R., ROSA-ZURERA, M., JARABO-AMORES, M. P., et al. Coherent detection of Swerling 0 targets in sea-ice Weibulldistributed clutter using neural networks. IEEE Transactions on Instrumentation and Measurement, 2010, vol. 59, no. 12, p. 3139-3151. DOI: 10.1109/TIM.2010.2047579

[21] SARIKAYA, T. B., SOYSAL, G., EFE, M., et al. Sea-land classification using radar clutter statistics for shore-based surveillance radars. In International Conference on Radar Systems (Radar 2017). Belfast (UK), 2017, p. 1-4. DOI: 10.1049/cp.2017.0488

[22] PAN, M., CHEN, J., WANG, S., et al. A novel approach for marine small target detection based on deep learning. In IEEE 4th International Conference on Signal and Image Processing (ICSIP). Wuxi (China), 2019, p. 395-399. DOI: $10.1109 /$ SIPROCESS.2019.8868862
[23] ZHU, L., XIONG, G., YU, W. Radar HRRP group-target recognition based on combined methods in the background of sea clutter. In International Conference on Radar. Brisbane (QLD, Australia), 2018, p. 1-6. DOI: 10.1109/RADAR.2018.8557334

[24] MAESTRE, DEL-REY, N., MATA-MOYA, D., JARABOAMORES, P., et al. Single MLP-CFAR for a radar Doppler processor based on the ML criterion. Validation on real data. In European Radar Conference. Paris (France), 2015, p. 53-56. DOI: 10.1109/EuRAD.2015.7346235

[25] CHEN, X., GUAN, J., HE, Y., ZHANG, J. Detection of low observable moving target in sea clutter via fractal characteristics in fractional Fourier transform domain. IET Radar, Sonar \& Navigation, 2013, vol. 7, no. 6, p. 635-651. DOI: 10.1049/iet-rsn.2012.0116

[26] DROSOPOULOS, A. Description of the OHGR Database. Technical Note 94-14, National Defence Canada, 1994, 42 pages. [Online] Cited 2019-03-13. Available at: https://apps.dtic.mil/dtic/tr/fulltext/u2/a290146.pdf

[27] BOCQUET, S. Parameter estimation for Pareto and K distributed clutter with noise. IET Radar, Sonar \& Navigation, 2015, vol. 9, no. 1, p. 104-113. DOI: 10.1049/iet-rsn.2014.0148

[28] MEZACHE, A., CHALABI, I., SOLTANI, F., et al. Estimating the Pareto plus noise distribution parameters using non-integer order moments and $[\mathrm{z} \log (\mathrm{z})]$ approaches. IET Radar, Sonar \& Navigation, 2016, vol. 10, no. 1, p. 192-204. DOI: 10.1049/iet-rsn.2015.0170

[29] FELLER, W. An Introduction to Probability Theory and its Applications. Vol. II. 2nd ed. New York (USA): John Wiley \& Sons Inc., 1971. ISBN: 978-0-471-25709-7

[30] TRAVEN, H. G. C. A neural network approach to statistical pattern classification by "semiparametric" estimation of probability density functions. IEEE Transactions on Neural Networks, 1991, vol. 2, no. 3, p. 366-377. DOI: 10.1109/72.97913

[31] ZEEVI, A., MEIR, R. Density estimation through convex combinations of densities: Approximation and estimation bounds. Neural Networks, 1997, vol. 10, no. 1, p. 99-109. DOI: 10.1016/S0893-6080(96)00037-8

[32] GHAHRAMANI, Z. Proceedings of the 1993 Connectionist Models Summer School. Hillsdale, NJ (USA): Lawrence Erlbaum Associates, 1994. (Solving inverse problems using an EM approach to density estimation.) ISBN: 9780805815900

[33] DEMPSTER, A. P., LAIRD, N. M., RUBIN, D. B. Maximum likelihood from incomplete data via the EM algorithm. Journal of the Royal Statistical Society: Series B, 1977, vol. 39, p. 1-38. DOI: 10.1111/j.2517-6161.1977.tb01600.x

[34] GLASSER, L., KOHL, K., KOUTSCHAN, C., et al. The integrals in Gradshteyn and Ryzhik. Part 22: Bessel-K functions. Scientia, Series A: Mathematical Sciences, 2012, vol. 22, p. 129-151. ISSN: 0716-8446

[35] ZOROVIC, D., MOHOVIC, R., MOHOVIC, D. Towards determining the length of the wind waves of the Adriatic Sea (In Croatian). Naše more, 2003, vol. 60, no. 3-4, p. 145-150. ISSN: 0469-6255

[36] BALLERI, A., NEHORAI, A., WANG, J. Maximum likelihood estimation for compound-Gaussian clutter with inverse gamma texture. IEEE Transactions on Aerospace and Electronic Systems, 2007, vol. 43 , no. 2, p. 775-779. DOI: 10.1109/TAES.2007.4285370

[37] PULFORD, G. W., LA SCALA, B. F. Multihypothesis Viterbi data association. Algorithm development and assessment. IEEE Transactions On Aerospace And Electronic Systems, 2010, vol. 46, no. 2, p. 583-609. DOI: 10.1109/TAES.2010.5461643 
[38] ROY, L. P., KUMAR, R. V. R. Accurate K-distributed clutter model for scanning radar application. IET Radar, Sonar and Navigation, 2010, vol. 4, no. 2, p. 158-167. DOI: 10.1049/iet-rsn.2009.0108

[39] NEYMAN, J., PEARSON, E. S. On the problem of the most efficient test of statistical hypotheses. Philosophical Transactions of the Royal Society of London, 1933, Series A, p. 289-337. DOI: $10.1098 /$ rsta.1933.0009

[40] BREKKE, E. F., HALLINGSTAD, O., GLATTETRE, J. H. Target tracking in heavy-tailed clutter using amplitude information. In 12th International Conference on Information Fusion. Seattle, WA (USA), 2009, p. 2153-2160. ISBN: 978-0-9824-4380-4

[41] WEINBERG, G. V., HOWARD, S. D., TRAN, C. A Bayesianbased CFAR detector for Pareto Type II clutter. In International Conference on Radar. Brisbane (QLD, Australia), 2018, p. 1-6. DOI: $10.1109 /$ RADAR.2018.8557282

[42] WEINBERG, G. V., BATEMAN, L., HAYDEN, P. Constant false alarm rate detection in Pareto Type II clutter. Digital Signal Processing, 2017, vol. 68, p. 192-198. DOI: 10.1016/j.dsp.2017.06.014

[43] ESI GROUP. Scilab: Free and Open Source Software for $\mathrm{Nu}$ merical Computation. [Online] Cited 2019-11-17. Available at: https://www.scilab.org

[44] SUSE LLC. Reference: openSUSE Leap 15.1. [Online] Cited 2019-11-19. Available at: https://doc.opensuse.org/documentation /leap/reference/book.opensuse.reference_color_en.pdf

[45] INTEL. Intel Core i5-3210M Processor. [Online] Cited 201911-19. Available at: https://ark.intel.com/content/www/us/en/ark/ products/65708/intel-core-i5-3210m-processor-3m-cache-up-to-310-ghz-bga.html

[46] SWERLING, P. Probability of Detection for Fluctuating Targets. Research Memorandum RM-1217, Santa Monica, CA (USA): RAND Corporation, 1954, 45 pages. [Online] Cited 2019-03-22. Available at: https://www.rand.org/pubs/research_memoranda/RM1217.html

[47] HAZEWINKEL, M. (Ed.) Encyclopaedia of Mathematics: Supplement Volume II. Dordrecht (Nederlands): Kluwer Academic Publishers, 2012. ISBN: 978-90-481-5378-7

[48] JIANG, H., YI, W., CUI, G., et al. Knowledge-based trackbefore-detect strategies for fluctuating targets in K-distributed clutter. Sensors, 2016, vol. 16, no. 19, p. 7124-7132. DOI: $10.1109 /$ JSEN.2016.2597320

\section{About the Authors ...}

Bojan VONDRA received his Dipl.Ing. degree in Electrical Engineering from the University of Zagreb, Faculty of Electrical Engineering and Computing (FER), Zagreb, Croatia, in 1990. From 1992 to 2001 he is with the Center for
Marine Electronics (PCE), Split, engaged in maintenance of naval marine radar systems. From 2001 to 2004 he is with Radio Communication Devices branch of Siemens, working on radio-frequency front-ends for Bluetooth devices. Since 2004 he is with the Center for Marine Electronics again, involved in fusion of coastal radar systems and algorithms for radar tracking of small targets. In 2013 he was promoted to Manager of Radar Department in the same company. He is currently pursuing $\mathrm{Ph} . \mathrm{D}$. degree in electrical engineering from the University of Zagreb, Faculty of Electrical Engineering and Computing (FER), Zagreb, Croatia.

Davor BONEFAČIĆ received his Dipl.Ing., Mr.Sc. and Dr.Sc. degrees in Electrical Engineering from the University of Zagreb, Faculty of Electrical Engineering and Computing (FER), Zagreb, Croatia, in 1993, 1996 and 2000, respectively. In 1993 he joined the Department of Wireless Communications at the Faculty of Electrical Engineering and Computing in Zagreb. Today he is Full Professor at the same Department. In 1996 he was a visiting researcher at the Third University of Rome, Rome, Italy. His research interests are in the field of small and integrated antennas, textile antennas and waveguiding structures, and on-body communications. He published one book chapter and more than 100 scientific papers in journals and conference proceedings. In the period 2003-2014, he served as a member of the editorial board of Radioengineering journal. He was the editor of twelve international conference proceedings in printed and electronic form. He is co-author of three university textbooks. His teaching activity includes several subjects on microwave engineering, RF systems, antennas and radars at graduate and doctoral studies. His professional activities are in the field of EMC, EMI estimation and suppression, EM field measurement and estimation of human exposure to EM fields. He authored and co-authored more than 35 professional and technical papers for government entities and companies. He is a member of the Croatian Academy of Engineering (HATZ) and he was elected Secretary of the Dept of Communication Systems of the Academy for the terms 2013-2017 and 2017-2021. He is a senior member of IEEE and has served several terms as chapter chair, vice-chair and treasurer in the MTT and AES/GRS Chapters of the IEEE Croatia Section. He received the silver plaque "J. Lončar" from FER for outstanding master thesis in 1996. In 2009 he received the "Rikard Podhorsky" award from the Croatian Academy of Engineering (HATZ) for excellence in scientific and professional achievements. 